\title{
3D FINITE ELEMENT STRESS ANALYSIS OF DIFFERENT ABUTMENT MATERIALS IN SCREW, IMPLANT AND CORTICAL BONE
}

\author{
Amany M. Korsel*
}

\begin{abstract}
Purpose: To give an idea of different implant-abutment material behavior by evaluation the stress distribution of material type on surrounding structures of single implant- supported restorations.

Materials and Methods : A 3D Finite Analysis method used for evaluate the distribution of stress in implants were examined using different abutment materials: Titanium(Ti) - (Implant Blue Sky, Bredent, Senden, Germany), Zirconium(Zr) (Implant Blue Sky, Bredent, Senden, Germany), High Performance Polymer (BioHPP)-( Sky elegance abutment, Bredent, Senden, Germany). Fiber reinforced composite(FRC)( World Headquarters sigma-aldrich.) Loading applied and as loading five nodes top an monolithic ceramic restoration. Mises and deflection values through screw and implant and cortical bone were calculated.
\end{abstract}

Results : The results of the analysis showed that the abutment material affect stress distributions where the least stresses created on bone with BioHPP abutment (16.5Mpa) followed with FRC abutment $(17.3 \mathrm{Mpa})$. While its effect on screw showed clear differences, the least stresses created with Zirconia abutment $(67.9 \mathrm{Mpa})$ followed by Titanium abutment $(85.29 \mathrm{Mpa})$ then FRC (117.32Mpa) finally abutment. These showed BioHPP FRC abutments nearly have very close and less effort bone. The highest stresses on implant showed with FRC and BioHPP abutments as follow $(142.4,122.4 \mathrm{Mpa})$ while the least stresses with Zirconium and Titanium abutments $(126.5,137.45 \mathrm{Mpa})$.

Conclusion: BioHPP and FRC abutment transmit less stress on the bone than Titanium and Zirconium . KEY WORDS : implant abutment,BioHPP , FRC,Stress analysis

KEY WORDS: implant abutment,BioHPP , FRC,Stress analysis

\footnotetext{
* Ass,Prof, Fixed Prosthodontics Department, Faculty of Dentistry, Tanta University, Egypt
} 


\section{INTRODUCTION}

Dental implants-supported restorations are con sidered a reliable treatment modality for restoring lost teeth. Implant failures after delivery of prosthesis are mainly attributed to biomechanical complications influenced by the load transferred through the bone-implant-prosthesis complex ${ }^{[1]}$.

Implant -abutments are, usually fabricated from commercially available pure titanium since it has biocompatibility and mechanical properties. Although production methods andtitanium implantabutments designs have been developed, the metallic gray color still shine through the thin mucosa and impair the esthetic outcomes. Even when titanium abutments are placed subgingivally, their gray zone causes an unnatural bluish appearance on the soft tissues $^{[2]}$. An esthetic implant - abutments had been suggested to improve the esthetic appearance of implant ceramic restorations ${ }^{[3]}$.

It is suggested that several factors may influence load distribution on implants surrounding structure such as geometry, number, length, diameter, and angulations of implants, and location of the implant. The relation between implant- abutment complex design and load distribution at the bone-implant interface is important to be understanding ${ }^{[4]}$.

A ceramic implant -abutment material that has better physical properties as zirconia abutment improves the gingival discolorations that occur for the patients, who have thin soft tissue. None the less, the type and structure of the implant-abutment connection might have a substantial on stability fixation brittle ceramic abutments.

None the less, the type and structure of the implant-abutment connection might have a substantial influence on the stability and fixation of brittle ceramic abutments. Designs of implants include different types of implant-abutment connections. Zirconia abutments combined with titanium bases have much higher fracture strengths than pure zirconia abutments, and two-piece zirconia abutments may be used for single-implant restorations ${ }^{[4-7]}$.
Functional occlusal load and overloading of dental implants during mastication has been extensively studied and discussed. There are other factors, affect the load transfer at the bone-implant interface such as the type of loading, material properties of the implant, abutment and prosthesis, surface structure, quality and quantity of the surrounding bone, and nature of the Osseointegration ${ }^{[5]}$. For dental applications, BioHPP materials also have been developed to improve the mechanical properties and colors of the restorations.

They are fabricated either with CAD / CAM or with compression-molding with Titanium adhesive bases that have been used . Polyetheretherketone (PEEK) is a high performance thermoplastic polymer, and PEEK based materials have been used in orthopedics, medicine and dentistry because of their features such as biocompatibility, elastic modulus comparable to bone and reduced stress shielding.

On the other hand, high performance polymers like modified and reinforced PEEK that is based with $20 \%$ fillers been used the of and Its of is similar to bone, and it is highly biocompatible with soft tissues ${ }^{[8]}$. The use of this white polymeric material has the advantages of preventing allergic reactions, good abrasion resistance, good polishing ability and low plaque retention.

For dental applications, ceramic reinforced PEEK materials (BioHPP) have been developed to improve the mechanical properties and colors of the restorations. Today there are different dental implant abutment materials available. A major challenge for clinicians today is understanding the biologic response to each material, as well as the best indication for using each of the different types.

Proper treatment planning for implant placement and careful crown fabrication with optimal cusp inclination can normally prevent implant overload ${ }^{[9]}$. The excessive bending moments may cause stress concentration and micro-fractures in alveolar bone and even implant fractures. Additionally, 
the modulus of elasticity of the bone and the high stiffness metallic implant are mismatched so, this induces the stress-shielding (3) effect, which means the implant does not strain and load the bone physiologically similarly as the absence of the implant. This causes loss of bone and bone contact $^{[10]}$.

So the concept of using a material with similar modulus of elasticity to bone has become an object of interest. By lowering the modulus of elasticity, the mechanical properties of the implant can better match the properties of bone which further provides mechanical stimulus to the bone-implant interaction. A relatively new group of materials in dental or medical applications as Fiber reinforced composites (FRC) are investigated ${ }^{[10]}$.

Their use is growing in many dental applications including use in implant-supported prostheses. The Finite Element Analysis (FEA) is a numerical technique useful for problems with complicated geometries, loadings, and material properties where analytical solutions cannot be obtained ${ }^{[11-14]}$. FEA has become an increasingly useful tool in the past few decades.

Additionally, it is used for determination of the stress distribution inside of the bone and various dental implant designs during function; such measurements are impossible to detected in vivo $^{[14]}$. The Finite Element Stress Analysis (FEA) uses virtual models to simulate and test the stress distribution of complex structures. According to FEA studies, this method enables the investigation of mechanical problems, dividing the elementproblem into many smaller and simpler elements to create a mesh of elements and to solve the problem by using mathematical functions.

This system enables researchers to apply different loadings and to obtain the displacement and the stress levels on the tooth, prosthesis, implant, and bone. The mechanical modeling of the structures can be performed in 2 or 3 dimensions. The 3 dimensional analysis allows for the development of models that are more true to real life and have complex geometry thereby creating more consistent results. The researchers used FEA to know how the stresses are distributed in the implants

\section{MATERIAL AND METHODS}

\section{Geometrical model:}

The "Premolar crown" has too complicated geometry, therefore a three dimensional scanner (Figure 1) was utilized for its modeling, Roland Model 15 to produce cloud of points or triangulations to be trimmed before using in any other application . An intermediate, software was required (Rhinoceros version to out crown that was to element as file format.

Bone was and as cylinders that consist of two coaxial cylinders. The inner represents spongy that the internal space of the other cylinder (shell of $1 \mathrm{~mm}$ thickness), which represents cortical bone. The implant-abutment complex was modeled in 3D using the commercial general-purpose CAD-CAM software ${ }^{[5]}$.

\section{Materials:}

The Youngs' Modulus (MPa) and Poissons' Ratio of the used materials in this study were assumed to be isotropic, homogenous, and linearly elastic were listed in Table 1

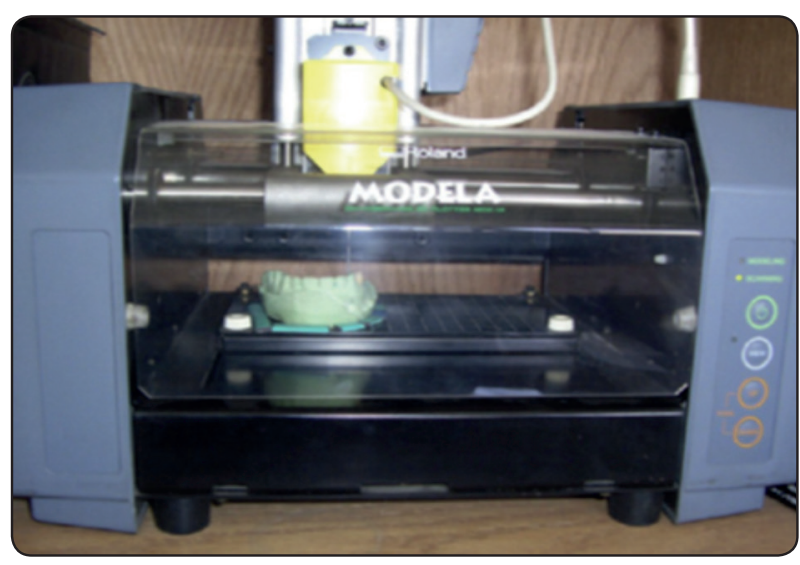

Fig. (1) Crown during 3D scanning and resulted geometry 
TABLE (1) List of the used materials' properties in analysis

\begin{tabular}{|l|l|l|l|l|}
\hline & Volumes & Material & Youngs' Modulus (MPa) & Poissons' Ratio \\
\hline $\mathbf{1}$ & Abutment & Titanium & 110,000 & 0.35 \\
& & Zirconia & 219,000 & 0.33 \\
& & BioHPP & 18,000 & 0.37 \\
& & FRC & 24,600 & 0.16 \\
\hline $\mathbf{2}$ & Screw & Titanium & 111,000 & 0.32 \\
\hline $\mathbf{3}$ & Fixture & Titanium & 110,000 & 0.33 \\
\hline $\mathbf{4}$ & Cortical Bone & Bone & 13,700 & 0.31 \\
\hline $\mathbf{6}$ & Crown & Monolithic lithium ceramic & 62,000 & 0.31 \\
\hline
\end{tabular}

\section{Meshing:}

All model parts were meshed (as presented in Figure X.4), by 8 nodes brick element Solid 1856 which has three translation degrees of freedom in the global axes directions. The model was subjected to Vertical loading of $200 \mathrm{~N}$ at lingual slope .

\section{Analysis:}

Set of linear static analyses was carried out simulating the following cases;

Vertical loading 200N applied at lingual cusp slope of the abutments; Titanium ,Zirconia, BioHPP and FRC Solid modeling and FEA were carried out on a personal computer, Intel Pentium Core to Duo, processor 3.2GHz, 4.0GB RAM. The meshing software was ANSYS version 14.0

\section{RESULTS}

Four runs were carried out by the described numerical model. Each run was performed with a different abutment material and each one subjected to vertical and oblique loading. Von Misses stresses on bone, implant, screw , cement and the crown of the studied system were obtained. A sample of the stress analysis results obtained shown for the screw, implant and for the cortical bone in. Fig 2, Fig 3, and Fig 4 ,Von Misses analysis in Mpa of four different abutment materials within the screw implant and cortical bone are shown in Table2.

TABLE (2) Von Misses Vertical load of different abutments in Mps

\begin{tabular}{|l|l|l|l|}
\hline Abutment Materials & Screw & Implant & Bone \\
\hline Titanium abutment & 85.29 & 137.45 & 19.50 \\
\hline Zirconia abutment & 67.94 & 126.55 & 19.87 \\
\hline BioHPP abutment & 223.3 & 122.45 & 16.55 \\
\hline FRC abutment & 117.3 & 142.4 & 17.3 \\
\hline
\end{tabular}

Von Misses analysis of four different abutment materials used in this study showed different effects on the variables, its effect on bone there was no clear difference but the least stresses created on bone with BioHPP abutment followed with FRC abutment. While its effect on screw showed clear differences, the least stresses created with Zirconia abutment followed by Titanium abutment then FRC and finally BioHPP abutment . .these results showed that BioHPP and FRC abutments nearly have very close effect on the screw and bone. The highest stresses on implant showed with FRC and BioHPP abutments .while the least stresses with zirconia and titanium abutments . 

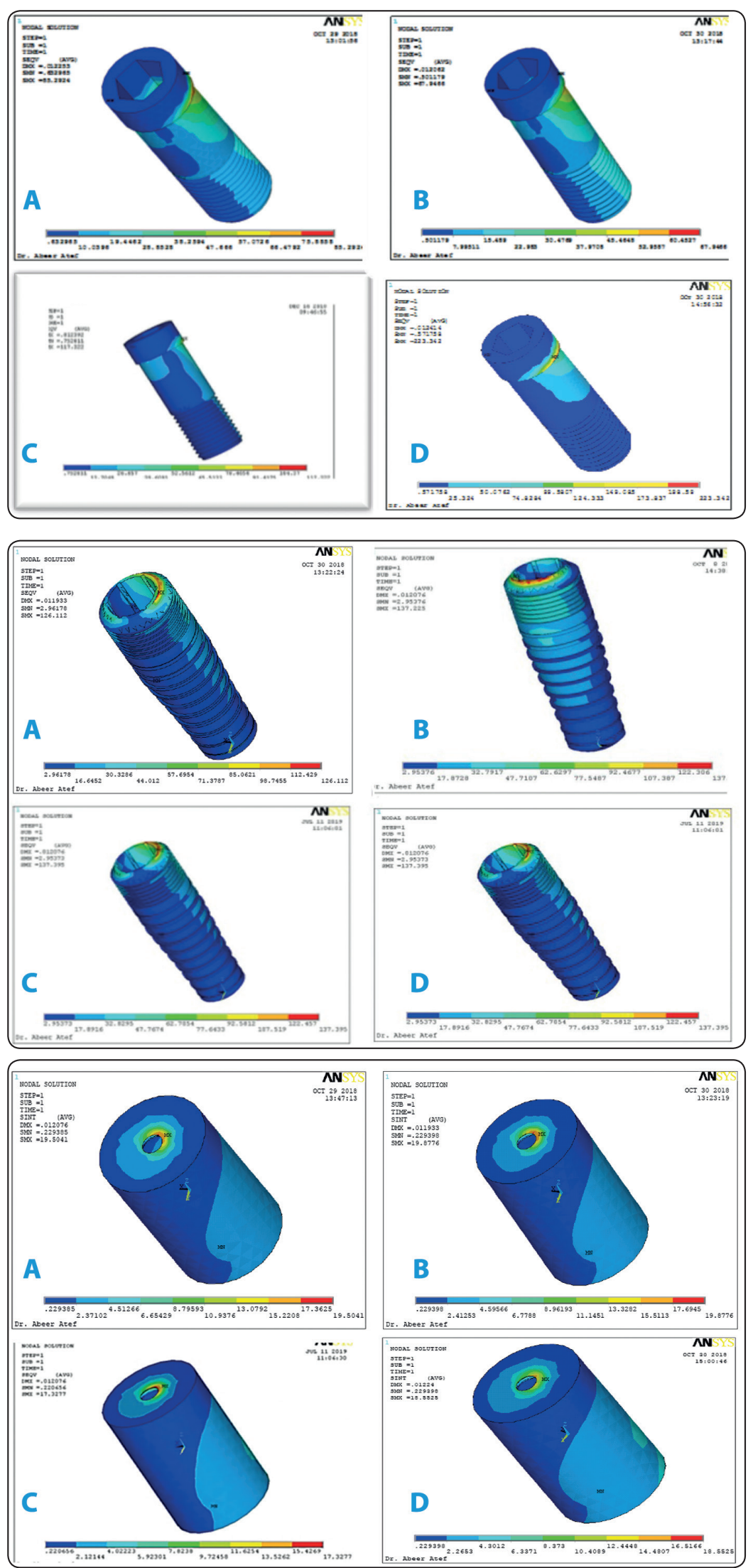

Fig. (2) Von Misses analysis on screw in different abutment materials (a) Titanium abutment (b) Zirconium abutment. (c) FRC abutment (d) BioHPP abutment.

Fig. (3) Von Misses analysis on implant in different abutment materials: (a) Titanium abutment (b) Zirconium abutment (c) FRC abutment. (d) BioHPP abutment,

Fig. (4) Von Misses analysis on bone in different abutment materials(a) Titanium abutment (b) Zirconium abutment. (c) FRC abutment (d) BioHPP abutment. 


\section{DISCUSSION}

After stage I surgery and prosthetic conclusion, the major cause of failure has been attributed to implant overload ${ }^{[2,3]}$. Although this question remains unanswered, there is a general agreement that a well-planned and executed prosthesis is essential to avoiding excessive forces on bone and implant components. Since the occlusal load will be transferred to the implants and subsequently to the bone, it is believed that the biomechanics of the implant-supported prosthesis play an important role in the longevity of the bone around dental implants.

${ }^{[4]}$. Under load, bone tissue undergoes a remodeling process, which ultimately influences the long-term function of a dental implant system ${ }^{[5]}$. The idea is to keep stresses below the failure stress of the bone ${ }^{[4-7]}$.

To be clear in clinical variables how affect stress distribution, this facilitates the selection of optimal prosthesis design and fabrication and may lead to a decrease in mechanical failures. In this study, the variations present in an implant-supported prosthesis were analyzed by a 3-dimensional finiteelement method.

The distribution of stresses around dental implants and how the loads are distributed must be known due to the lack of periodontal ligament may affect the stress distribution. This can lead to bone loss if high stresses are concentrated on the boneimplant interface ${ }^{[7]}$.

In the present study, we analyzed the stress distribution (Von Misses stress analyses) on different variables (screw, implant fixture, bone) using four different abutment materials, our results in agree with Yildirim et $\mathrm{al}^{[8]}$. reported that zirconia abutments obtained values that were more than two times higher than those of the alumina ones.

In this study zirconia abutment showed higher stress concentrations on bone than the BioHPP and FRC which considered resilient materials like
alumina.While these materials concentrate higher forces througth the screw and implant as a reverse to the titanium and zirconia abutments.

Sundh and Sjo"gren ${ }^{[9]}$ evaluated the resistance of implant-supported CAD/CAM-fabricated restorations made out of zirconia or manually shaped from reinforced alumina. All the ceramic abutments and copies showed values that were equal or superior to those of the control and exceeded the recorded value,

Another important aspect to discuss is the elastic modulus of the abutment materials used.A material with smaller elastic modulus presents smaller flexural resistance, whereas substructures made with rigid metal alloys undergo smaller deformation because they are less susceptible to fatigue[10]. Therefore in this study using BioHPP and FRC with less elastic modulous than zirconia and titanium abutment which distribute less stress on bone .

\section{REFERENCE}

1. Maminskas J, Puisys A, Kuoppala R, Raustia A, Juodzbalys G. The prosthetic influence and biomechanics on periimplant strain: a systematic literature review of finite element studies. J Oral Maxillof. Res. 2016;7:e4.

2. Gass SI. Decision-adding models: validation, assessment and related issues for policy analysis. Oper Res. 1983; 31:603-631.

3. Tada S. Influence of implant design and bone quality on stress/strain distribution in bone around implants: a 3-dimensional finite element analysis. Int J Oral Maxillofac Implants. 2003; 18:357-68.

4. Sevimay M, Turhan F, Kilic, arslan MA, Eskitascioglu G. Three-dimensional finite element analysis of the effect of different bone quality on stress distribution in an implantsupported crown. J Prosthet Dent 2005; 93: 227-34.

5. Arenal AA, Mori LS,Gonzalez IG and Gago A. Stress distribution in the abutment and retention screw of a single implant supporting a prosthesis with platform switching. Int J of Maxillof. Implants 2013; 28: 112-21.

6. Cardosoa M,Corazzaa PH, Clarob CA, Borgesa A, Bottinoa MA and Juniora LN. Stress distribution around implants 
with abutments of different materials: a comparison of photoelastic, strain gage and finite element analyses. $\mathrm{J}$ of Dental Science 2015; 30:132-7.

7. Galvão G H, Grossi G A, Zielak J C,et al,. Influence of Metal and Ceramic Abutments on the Stress Distribution Around Narrow Implants: A Photoelastic Stress Analysis. Implant Dent 2016; 25: 499-503.

8. Yildirim M, Fischer H, Marx R, Edelhoff D. In vivo fracture resistance of implant-supported all-ceramic restorations. J Prosthet Dent 2003; 90: 325-331.

9. Sundh A, Sjo“ gren G. A study of the bending resistance of implant-supported reinforced alumina and machined zirconia abutments and copies. Dent Mater 2008; 24:611617.

10. Suedam V, Souza EA, Moura MS, et al.Effect of abutment's height and framework alloy on the load distribution of mandibular cantilevered implant supported prosthesis. Clin Oral Implants Res. 2009; 20:196-200.

11. Saadet Sağlam Atsü, M.Emin Aksan, ,Ali Can Bulut, ; Fracture Resistance of Titanium, Zirconia, and Ceramic-
Reinforced Polyetheretherketone Implant Abutments Supporting CAD/CAM Monolithic Lithium Disilicate Ceramic Crowns After Aging. Int J Oral Maxillof Implant 2019 ; May, 34(3):622-30

12. Elsayed Adham, Farrag Gasser, Sad Chaar Mohamed,et al:Influence of Different CAD/CAM Crown Materials on the Fracture of Custom-Made Titanium and Zirconia Implant Abutments After Artificial Aging . Int J Prosthodont 2019;32:91-96

13. El-S'adany A.F, Masoud G.E ,. Kamel M.S, Korsel A.M. Fracture resistance of all ceramic crowns supported by zirconia and alumina versus titanium implant abutments. Tanta Dental J 2013 ;Journal 10 ,103-111

14. El-Anwara Mohamed I, Fawzyb Usama M., Mohamadb Husien et al. Effect of different abutment materials of implant on stress distribution using three-dimensional finite element analysis Medical Research Journal 2013; Vol $12,(2)$ 